\title{
Simple model of the density of states in 1D photonic crystal
}

\author{
Adam Rudziński, Anna Tyszka-Zawadzka, Paweł Szczepański \\ Institute of Microelectronics and Optoelectronics, Warsaw University of Technology, Warsaw, \\ Poland
}

\begin{abstract}
In this paper, we present a simple, yet versatile, analytical model of one-dimensional photonic crystal (1D PC). In our theoretical model, we take into account direction of propagation and therefore do not neglect anisotropic nature of photonic crystals. We derive analytical expressions for mode spectrum and density of states in 1D photonic crystal. With those formulas, we obtain mode spectrum characteristics, which depict formation of photonic band gap and reveal properties of photonic crystals.
\end{abstract}

\section{INTRODUCTION}

Photonic crystals (PCs), proposed by Yablonovitch ${ }^{1}$ and John, ${ }^{2}$ represent a novel class of optical materials which allow to control the flow of electromagnetic radiation or to modify light-matter interaction. These artificial structures are characterized by one-, two- or three-dimensional arrangements of dielectric material which lead to the formation of an energy band structure for electromagnetic waves propagating in them. One of the most interesting features of photonic crystals is associated with the fact that PCs may exhibit frequency ranges over which ordinary linear propagation is forbidden, irrespective of direction. These photonic band gaps (PBGs) lend themselves to numerous diversified applications (in linear, nonlinear and quantum optics). For instance, PBG structures with line defects can be used for guiding light. Similarly, as it has been predicted and confirmed experimentally, photonic crystals allow to modify spontaneous emission rate due to the modification of density of quantum states. In particular, it is well known that the density of states grows at the edge of the photonic band gap of the PC. This allows us to predict a higher optical gain, but on the other hand a higher level of noise in light generated in PC-lasers operated at a frequency near the band gap.

It is our purpose here to present a simple, yet versatile, analytical model of one-dimensional photonic crystal. The investigated photonic crystal consists of $N$ elementary cells with alternating layers of different indices of refraction and different widths. Presented model takes into consideration direction of propagation and therefore does not neglect anisotropic nature of photonic crystals. We derive analytical expressions for mode spectrum and density of states that allow to analyze properties of one-dimensional photonic crystals, especially inhibition or amplification of spontaneous emission due to modification of density of states. The described model allows to examine basic physics behind PBG structures and as an analytical model - can provide an excellent starting point in numerical calculations for much more complicated structures.

\section{EFFECTIVE RESONANCE APPROACH TO PHOTONIC CRYSTALS}

The simplest case of a photonic crystal is one-dimensional photonic crystal. It is a structure built of alternating layers of linear, uniform and isotropic materials of two different refractive indices $n_{1}$ and $n_{2}$. Its section in $x z$ plane is shown on figure 11. The structure has rotational symmetry around $z$ axis, all layers extend towards infinity in $x$ and $y$ directions (direction $y$ is perpendicular to section shown on figure 1). We will consider a photonic crystal built of finite number of layers. A primitive cell in this case is a pair of subsequent layers with refractive indices $n_{1}$ and $n_{2}$. The number of primitive cells "right" from $z=0$ plane will be $N_{R}$ and to the "left" will be $N_{L}$, where directions "right" and "left" are in accordance with those on figure 1. We will introduce primitive cell index $m$ as depicted on the figure: layers with $z \in[0, \Lambda]$ constitute primitive cell with $m=0$, and the next (along with the $z$ axis) pair has $m=1$, the first one has $m=N_{R}-1$, the first primitive cell "left" from $z=0$ plane has $m=-1$ and the index of the last one is $m=-N_{L}$. We also assume (for simplicity), that the crystal is surrounded by a material with refractive index $n_{1}$.

The idea of the described method ${ }^{3 \mid 4}$ is presented on figure 2 . It aims to determine effective reflection from a part of photonic crystal on a layer's boundary and "replace" it with a mirror of the same reflection. Thus, 


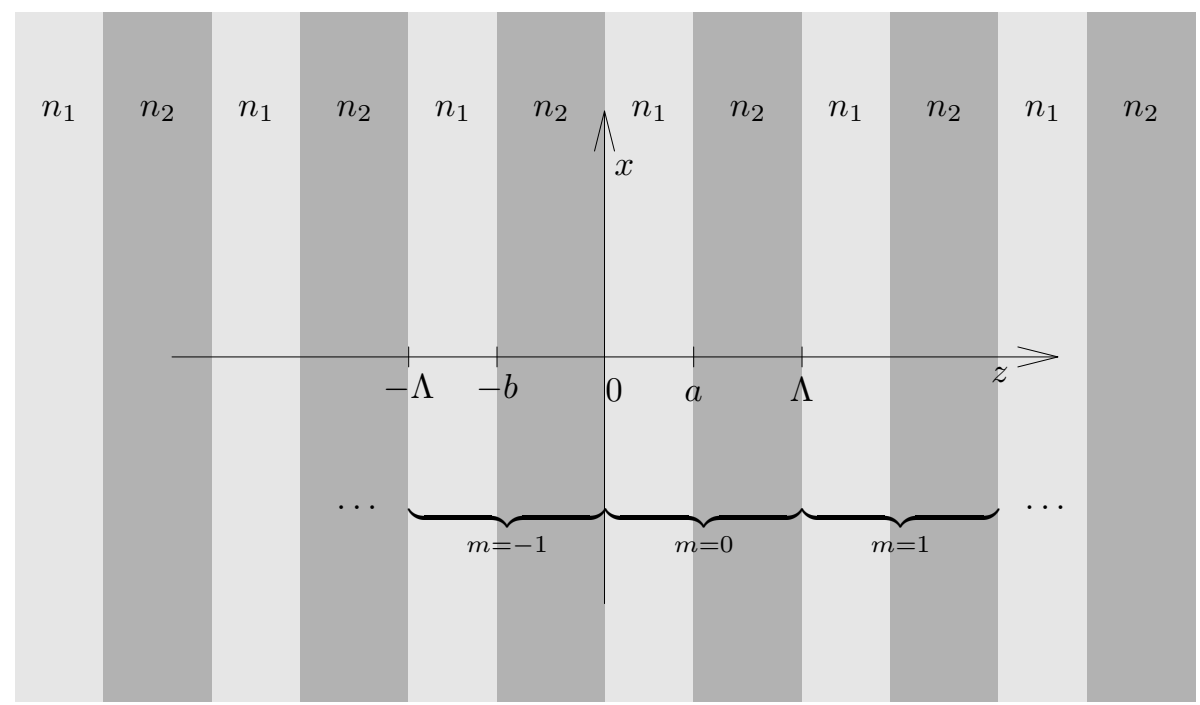

Figure 1. Structure of one-dimensional photonic crystal (section).

we can say, that chosen crystal's layer in which the density of states is investigated, is inside an effective FP cavity. This method can describe properties of finite photonic crystals, and allows to examine changes of these properties in different layers of the crystal. In general case of photonic crystal, precise description of density of states without approximations requires difficult and time consuming calculations. However, for the simplest case of one-dimensional photonic crystal it is possible to follow the derivation given below in the further part of the paper.

In our model we assume that in each of layers of the photonic crystal Maxwell equations ${ }^{5}$ have solutions in form of plane-waves:

$$
\vec{E}=\vec{E}_{0} e^{i \vec{k} \vec{r}-i \omega t}
$$

with wave vector $\vec{k}$ bound with angular frequency $\omega$ by dispersion relation

$$
\vec{k}^{2}=\frac{n^{2} \omega^{2}}{c^{2}}
$$

where $c$ is speed of light in vacuum and $n$ is refractive index of the material of the layer. We can relate fields in different layers using continuity conditions, what is especially simple in case of one-dimensional photonic crystal. 6 In every $n_{1}$ layer we represent electric field as a superposition of coupled plane-waves:

$$
E_{m, 1, \epsilon}=a_{m, \epsilon} e^{i k_{x} x+i \beta_{1} z-i \omega t}+b_{m, \epsilon} e^{i k_{x} x-i \beta_{1} z-i \omega t} \quad, \quad z \in[m \Lambda, m \Lambda+a]
$$

and similarly in every $n_{2}$ layer:

$$
E_{m, 2, \epsilon}=c_{m, \epsilon} e^{i k_{x} x+i \beta_{2} z-i \omega t}+d_{m, \epsilon} e^{i k_{x} x-i \beta_{2} z-i \omega t} \quad, \quad z \in[m \Lambda+a,(m+1) \Lambda]
$$

where $\epsilon$ denotes polarization $T E$ (electric field perpendicular to plane of incidence) or $T M$ (electric field in plane of incidence) and

$$
\beta_{j}=\sqrt{\frac{n_{j}^{2} \omega^{2}}{c^{2}}-k_{x}^{2}} \quad, \quad j=1,2
$$

Continuity conditions lead to equation relating amplitudes in consecutive primitive cells:

$$
\left[\begin{array}{l}
a_{m+1, \epsilon} \\
b_{m+1, \epsilon}
\end{array}\right]=M_{m, \epsilon}\left[\begin{array}{l}
a_{m, \epsilon} \\
b_{m, \epsilon}
\end{array}\right]
$$




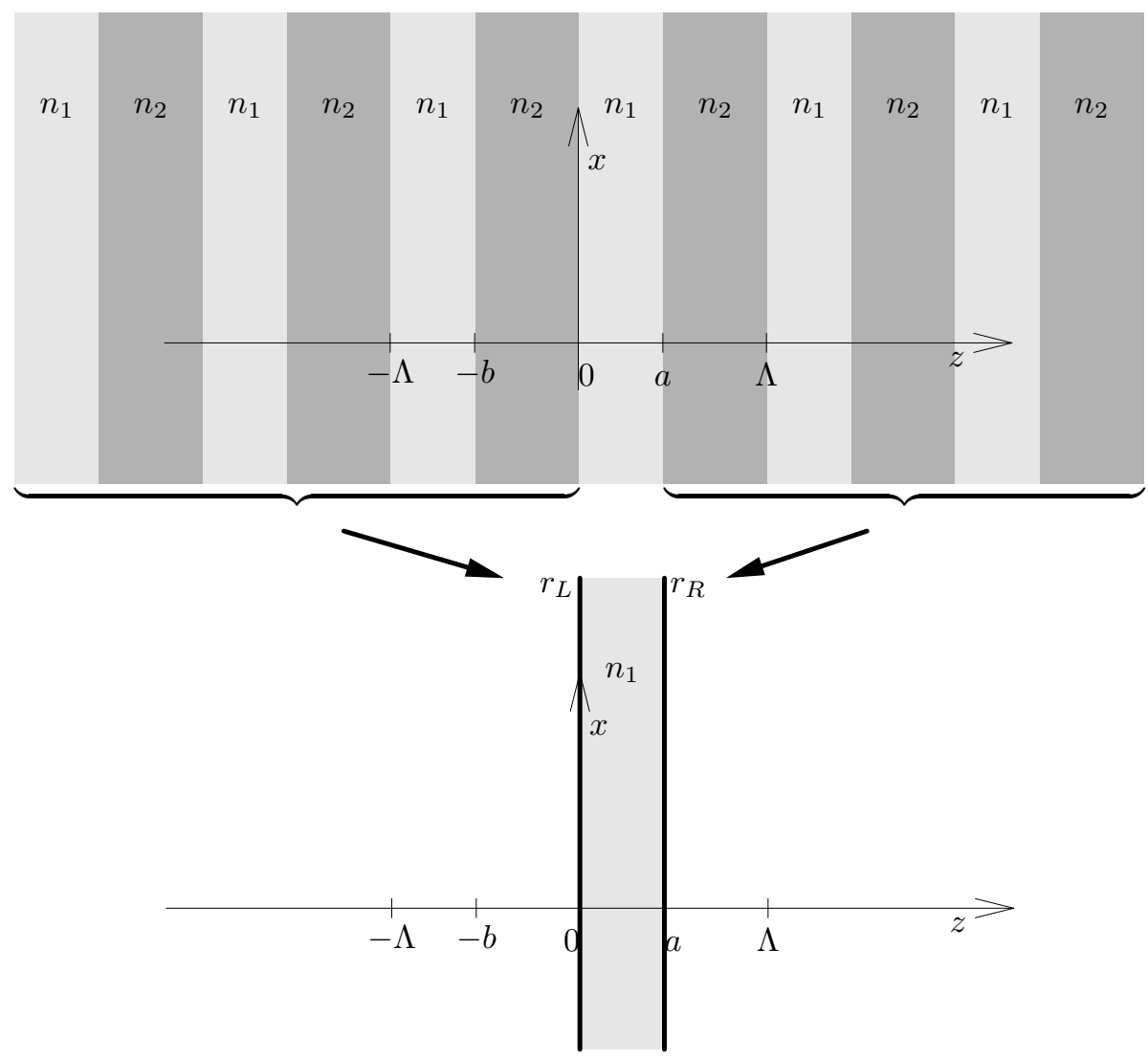

Figure 2. A layer of one-dimensional photonic crystal as a resonator.

where $M_{m, \epsilon}$ is translation matrix of $m$ th primitive cell. It is an obvious conclusion, that if amplitudes of planewaves outside the photonic crystal are indexed with $m=N_{R}$ for "right" and $m=-N_{L}-1$ for "left", then

$$
\begin{aligned}
& {\left[\begin{array}{l}
a_{N_{R}, \epsilon} \\
b_{N_{R}, \epsilon}
\end{array}\right]=\left(M_{N_{R}-1, \epsilon} M_{N_{R}-2, \epsilon} \ldots M_{1, \epsilon} M_{0, \epsilon}\right)\left[\begin{array}{l}
a_{0, \epsilon} \\
b_{0, \epsilon}
\end{array}\right]} \\
& {\left[\begin{array}{l}
a_{-N_{L}-1, \epsilon} \\
b_{-N_{L}-1, \epsilon}
\end{array}\right]=\left(M_{-N_{L}, \epsilon} M_{-N_{L}+1, \epsilon} \ldots M_{-2, \epsilon} M_{-1, \epsilon}\right)^{-1}\left[\begin{array}{l}
a_{0, \epsilon} \\
b_{0, \epsilon}
\end{array}\right]}
\end{aligned}
$$

with the amplitudes outside the crystal bound by external conditions. (77) and (8) are general relations, that allow to perform calculations for defected structures. For a perfect photonic crystal we have

$$
M_{m, \epsilon}=M_{m^{\prime}, \epsilon} \equiv M_{\epsilon} \quad, \quad m, m^{\prime} \in\left[-N_{L}, N_{R}-1\right]
$$

with

$$
M_{\epsilon}=\left[\begin{array}{cc}
A_{\epsilon} & B_{\epsilon} \\
C_{\epsilon} & D_{\epsilon}
\end{array}\right]
$$

where

$$
\begin{aligned}
& A_{T E}=e^{i \beta_{1} a}\left(\cos \left(\beta_{2} b\right)+\frac{i}{2}\left(\frac{\beta_{1}}{\beta_{2}}+\frac{\beta_{2}}{\beta_{1}}\right) \sin \left(\beta_{2} b\right)\right) \\
& B_{T E}=\frac{i}{2} e^{-i \beta_{1} a}\left(\frac{\beta_{2}}{\beta_{1}}-\frac{\beta_{1}}{\beta_{2}}\right) \sin \left(\beta_{2} b\right) \\
& C_{T E}=\frac{i}{2} e^{i \beta_{1} a}\left(\frac{\beta_{1}}{\beta_{2}}-\frac{\beta_{2}}{\beta_{1}}\right) \sin \left(\beta_{2} b\right) \\
& D_{T E}=e^{-i \beta_{1} a}\left(\cos \left(\beta_{2} b\right)-\frac{i}{2}\left(\frac{\beta_{1}}{\beta_{2}}+\frac{\beta_{2}}{\beta_{1}}\right) \sin \left(\beta_{2} b\right)\right)
\end{aligned}
$$


and

$$
\begin{aligned}
A_{T M} & =e^{i \beta_{1} a}\left(\cos \left(\beta_{2} b\right)+\frac{i}{2}\left(\frac{n_{2}^{2} \beta_{1}}{n_{1}^{2} \beta_{2}}+\frac{n_{1}^{2} \beta_{2}}{n_{2}^{2} \beta_{1}}\right) \sin \left(\beta_{2} b\right)\right) \\
B_{T M} & =\frac{i}{2} e^{-i \beta_{1} a}\left(\frac{n_{1}^{2} \beta_{2}}{n_{2}^{2} \beta_{1}}-\frac{n_{2}^{2} \beta_{1}}{n_{1}^{2} \beta_{2}}\right) \sin \left(\beta_{2} b\right) \\
C_{T M} & =\frac{i}{2} e^{i \beta_{1} a}\left(\frac{n_{2}^{2} \beta_{1}}{n_{1}^{2} \beta_{2}}-\frac{n_{1}^{2} \beta_{2}}{n_{2}^{2} \beta_{1}}\right) \sin \left(\beta_{2} b\right) \\
D_{T M} & =e^{-i \beta_{1} a}\left(\cos \left(\beta_{2} b\right)-\frac{i}{2}\left(\frac{n_{2}^{2} \beta_{1}}{n_{1}^{2} \beta_{2}}+\frac{n_{1}^{2} \beta_{2}}{n_{2}^{2} \beta_{1}}\right) \sin \left(\beta_{2} b\right)\right)
\end{aligned}
$$

As a result, (7) is simplified to

$$
\left[\begin{array}{l}
a_{N, \epsilon} \\
b_{N, \epsilon}
\end{array}\right]=M_{\epsilon}^{N}\left[\begin{array}{l}
a_{0, \epsilon} \\
b_{0, \epsilon}
\end{array}\right]
$$

(for more elegance the index $R$ is omitted). Matrix $M_{\epsilon}$ is unimodular, therefore is obeys the Chebyshev identity:

$$
M_{\epsilon}^{N}=\left[\begin{array}{cc}
A_{\epsilon} & B_{\epsilon} \\
C_{\epsilon} & D_{\epsilon}
\end{array}\right]^{N}=\left[\begin{array}{cc}
A_{\epsilon} U_{N, \epsilon}-U_{N-1, \epsilon} & B_{\epsilon} U_{N, \epsilon} \\
C_{\epsilon} U_{N, \epsilon} & D_{\epsilon} U_{N, \epsilon}-U_{N-1, \epsilon}
\end{array}\right]
$$

with

$$
U_{N, \epsilon}=\frac{\sin \left(N \eta_{\epsilon}\right)}{\sin \eta_{\epsilon}} \quad, \quad \eta_{\epsilon}=\arccos \frac{A_{\epsilon}+D_{\epsilon}}{2}
$$

what allows to simplify (19) even more. Relation (19) (or (7) and (8) in more general case) is a set of two linear equations. ${ }^{[4}$ Amplitudes $a_{N, \epsilon}$ and $b_{N, \epsilon}$ are bound by external conditions, in this case it is just

$$
b_{N, \epsilon}=0
$$

because we assume excitation in the photonic crystal (or at least on the other side of it) and there is no reflection in the infinity. Because $a_{0, \epsilon}$ is the amplitude of the wave incident on layer boundary in $z=a, b_{0, \epsilon}$ can be treated as reflected wave. Therefore, we can calculate reflection coefficient of the whole part of photonic crystal reflecting $a_{0, \epsilon}$ wave, it will be

$$
r_{\epsilon}=\frac{b_{0, \epsilon} e^{-i \beta_{1} a}}{a_{0, \epsilon} e^{i \beta_{1} a}}
$$

Knowing (19), (20) and (22) (and again marking index $R$ for "right" part of the crystal), we find that

$$
r_{R, \epsilon}=\frac{B_{\epsilon}}{D_{\epsilon}-\frac{\sin \left(\left(N_{R}-1\right) \eta_{\epsilon}\right)}{\sin \left(N_{R} \eta_{\epsilon}\right)}}
$$

Because the structure is symmetrical, we instantly have expression for "left" part reflection coefficient

$$
r_{L, \epsilon}=\frac{B_{\epsilon}}{D_{\epsilon}-\frac{\sin \left(\left(N_{L}-1\right) \eta_{\epsilon}\right)}{\sin \left(N_{L} \eta_{\epsilon}\right)}}
$$

(in general case of defected crystal it is not possible to obtain such a concise expression for at least one reflection coefficient).

Having calculated reflection coefficients, we treat the layer of photonic crystal as a resonator, with mirrors constituted by the remaining layers of the crystal and described by derived formulas. 


\section{MODE SPECTRUM AND DENSITY OF STATES IN A LAYER OF ONE-DIMENSIONAL PHOTONIC CRYSTAL}

Let operator $\hat{Q}$ relate electromagnetic plane-wave $\vec{E}^{e x}$ exciting the resonator to effective plane-wave $\vec{E}^{e f f}$ with the same wave vector, that appears in the resonator due to excitement:

$$
\vec{E}^{e f f}=\hat{Q} \vec{E}^{e x}
$$

We call a plane-wave $\vec{E}_{\epsilon}(\vec{k})$ with polarization $\epsilon$ and wave vector $\vec{k}$ a mode of the resonator if it satisfies eigenequation of $\hat{Q}$ :

$$
\hat{Q} \vec{E}_{\epsilon}(\vec{k})=\Lambda_{\epsilon}(\vec{k}) \vec{E}_{\epsilon}(\vec{k})
$$

If the resonator is excited with plane-wave $\vec{E}_{\epsilon}(\vec{k})$, effective distribution of electromagnetic field is superposition of plane-wave $\Lambda_{\epsilon}(\vec{k}) \vec{E}_{\epsilon}(\vec{k})$ with its coupled mode (i.e. plane wave with wave vector $\left[k_{x}, k_{y},-k_{z}\right]$ ), which appears because of reflections. Therefore, introduced definition is equivalent to "classical" definition of a mode.

We introduce mode spectrum $\rho_{\epsilon}(\vec{k})$, related to distribution of modes in wave vector domain. Thus, it can be used to calculate the number of modes $N(k)$ with propagation constants less than $k$ :

$$
N(k)=\sum_{\epsilon} \int_{0}^{k} d k^{\prime} k^{\prime 2} \int_{4 \pi} d \Omega^{\prime} \rho_{\epsilon}\left(\vec{k}^{\prime}\right)
$$

Differential of $N(k)$ is known as density of states. From (28) it can be found, that

$$
d N(k) d k=k^{2} d k \sum_{\epsilon} \int_{4 \pi} d \Omega \rho_{\epsilon}(\vec{k})
$$

Because operator $\hat{Q}$ can be used to find distribution of modes, mode spectrum $\rho_{\epsilon}(\vec{k})$ has to be proportional to its eigenvalues:

$$
\rho_{\epsilon}(\vec{k})=N_{\rho} \Lambda_{\epsilon}(\vec{k})
$$

From (29) we can calculate, that in free space, where $\Lambda_{\epsilon}(\vec{k})=1$,

$$
d N_{f s}(k) d k=k^{2} d k \sum_{\epsilon} \int_{4 \pi} d \Omega N_{\rho}=8 \pi N_{\rho} k^{2} d k
$$

It is a well known fact, that

$$
d N_{f s}(k) d k=\frac{k^{2}}{\pi^{2}} d k
$$

Comparing (31) and (32) we find the normalization constant $N_{\rho}$ :

$$
N_{\rho}=\frac{1}{(2 \pi)^{3}}
$$

Finally, mode spectrum is defined as

$$
\rho_{\epsilon}(\vec{k})=\frac{\Lambda_{\epsilon}(\vec{k})}{(2 \pi)^{3}}
$$

Mode spectrum depicts influence of a FP cavity on a plane-wave. It also contains information on anisotropy of the structure, and therefore is a magnitude more fundamental than density of states.

To find mode spectrum of a $n_{1}$ layer of one-dimensional photonic crystal we need to find operator $\hat{Q}$ for a FP resonator filled with medium with refractive index $n_{1}$, built of two parallel mirrors at distance $a$ from each other, 
with reflection coefficients $r_{R, \epsilon}$ and $r_{L, \epsilon}$. If the resonator is excited with a plane-wave with wave vector $\vec{k}$ and amplitude of electric field $E_{0}$ (this is a scalar problem), then amplitude of electric field of effective plane-wave with wave vector $\vec{k}$ can be found by summation of two infinite series: $\stackrel{3] 4}{[4}$

$$
E^{e f f}=E_{0}+\sum_{j=1}^{\infty} r_{R, \epsilon} r_{L, \epsilon} e^{2 i \beta a} E_{0}+\sum_{j=1}^{\infty} r_{R, \epsilon}^{*} r_{L, \epsilon}^{*} e^{-2 i \beta a} E_{0}
$$

where $\beta=\beta_{1}$ defined by (5) and ${ }^{*}$ means complex conjugation. Comparing to definition (26) we can conclude, that the operator $\hat{Q}$ for the resonator is:

$$
\hat{Q}=\frac{1-\left|r_{R, \epsilon} r_{L, \epsilon}\right|^{2}}{1+\left|r_{R, \epsilon} r_{L, \epsilon}\right|^{2}-2 \operatorname{Re}\left\{r_{R, \epsilon} r_{L, \epsilon} e^{2 i \beta a}\right\}}
$$

and from (34) we obtain the formula for mode spectrum of the photonic crystal's layer:

$$
\rho_{\epsilon}(\vec{k})=\frac{1}{8 \pi^{3}} \frac{1-\left|r_{R, \epsilon} r_{L, \epsilon}\right|^{2}}{1+\left|r_{R, \epsilon} r_{L, \epsilon}\right|^{2}-2 \operatorname{Re}\left\{r_{R, \epsilon} r_{L, \epsilon} e^{2 i \beta a}\right\}}
$$

To find density of states we can use definition (29). In spherical coordinates $\beta=k \cos \vartheta$, therefore

$$
d N(k)=k^{2} \int_{0}^{\pi} d \vartheta^{\prime} \sin \vartheta^{\prime} \int_{0}^{2 \pi} d \varphi^{\prime} \sum_{\epsilon} \frac{1}{8 \pi^{3}} \frac{1-\left|r_{R, \epsilon} r_{L, \epsilon}\right|^{2}}{1+\left|r_{R, \epsilon} r_{L, \epsilon}\right|^{2}-2 \operatorname{Re}\left\{r_{R, \epsilon} r_{L, \epsilon} e^{2 i a k \cos \vartheta^{\prime}}\right\}}
$$

Integration over $\varphi^{\prime}$ can be done, because of rotational symmetry of the crystal. Additionally substituting $u=\cos \vartheta^{\prime}$ we reach the following formula:

$$
d N(k)=\frac{k^{2}}{4 \pi^{2}} \int_{-1}^{1} d u \sum_{\epsilon} \frac{1-\left|r_{R, \epsilon} r_{L, \epsilon}\right|^{2}}{1+\left|r_{R, \epsilon} r_{L, \epsilon}\right|^{2}-2 \operatorname{Re}\left\{r_{R, \epsilon} r_{L, \epsilon} e^{2 i a k u}\right\}}
$$

The above expression is true for any resonator with two parallel mirrors. In particular, taking $r_{R, \epsilon}=r_{L, \epsilon}=0$ and $n_{1}=1$ we obtain expression for density of states in free space:

$$
d N_{f s}(k)=\frac{k^{2}}{\pi^{2}} d k
$$

If both $r_{L, \epsilon}$ and $r_{R, \epsilon}$ are constant, mode spectrum takes form of regularly distributed "peaks" (see figure 3 ).

\section{VARIATION OF MODE SPECTRUM IN A ONE-DIMENSIONAL PHOTONIC CRYSTAL}

Presented method of mode spectrum calculation allows to analyze its space dependence in photonic crystal and therefore also the crystals properties. For example, it is possible to examine the photonic band gap edge in different layers of the crystal (figure 4). It can be clearly seen, that in layers (sufficiently) close to the end of the photonic crystal full photonic band gap does not form. The reason of this behavior is that reflection coefficients $r_{L}$ and $r_{P}$ depend on number of layers surrounding selected layer. If this number is high, amplitude of reflection coefficient can be close to 1, while for low number of layers amplitude of reflection coefficient is significantly lower. In the second case, quality of resonator is low and its mode spectrum tends to be more similar to mode spectrum of free space. Therefore, formation of distinctive photonic band gap does not occur. This regularity can be as well observed on figure 5 which depicts mode spectrum (for perpendicularly incident plane-wave) in different layers of the crystal. Amplitude of mode spectrum at the edge of photonic band gap reaches highest values in the middle of the crystal, where amplitudes of reflection coefficients $r_{L}$ and $r_{P}$, along with effective resonator quality, have the highest values. Another interesting observation, is that photonic band gap in general is not symmetrical - amplitudes of mode spectrum at the edges of the gap are not equal. 


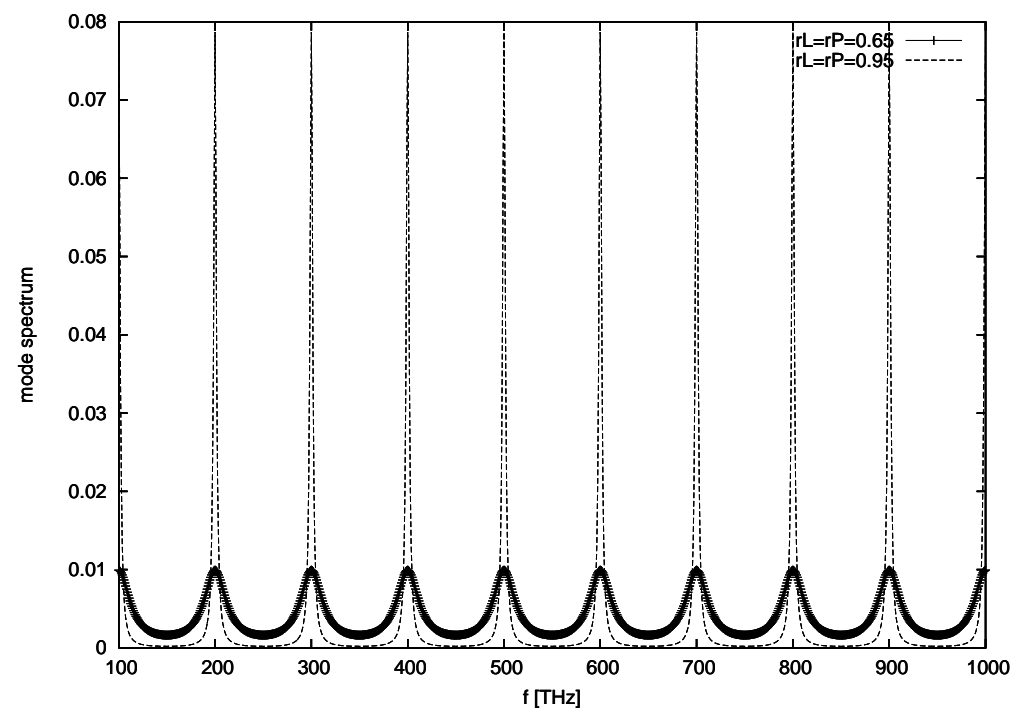

Figure 3. Modes of FP resonator with constant reflection coefficients of mirrors.
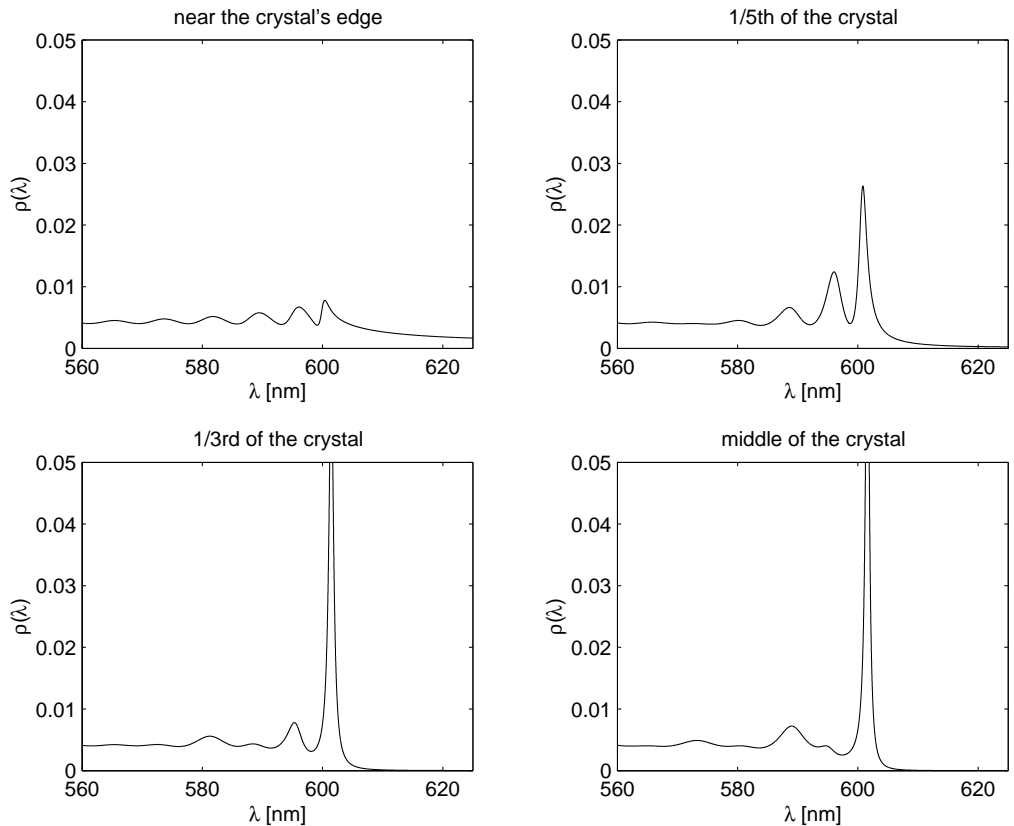

Figure 4. Edge of photonic band gap in different layers of a 1D photonic crystal. 


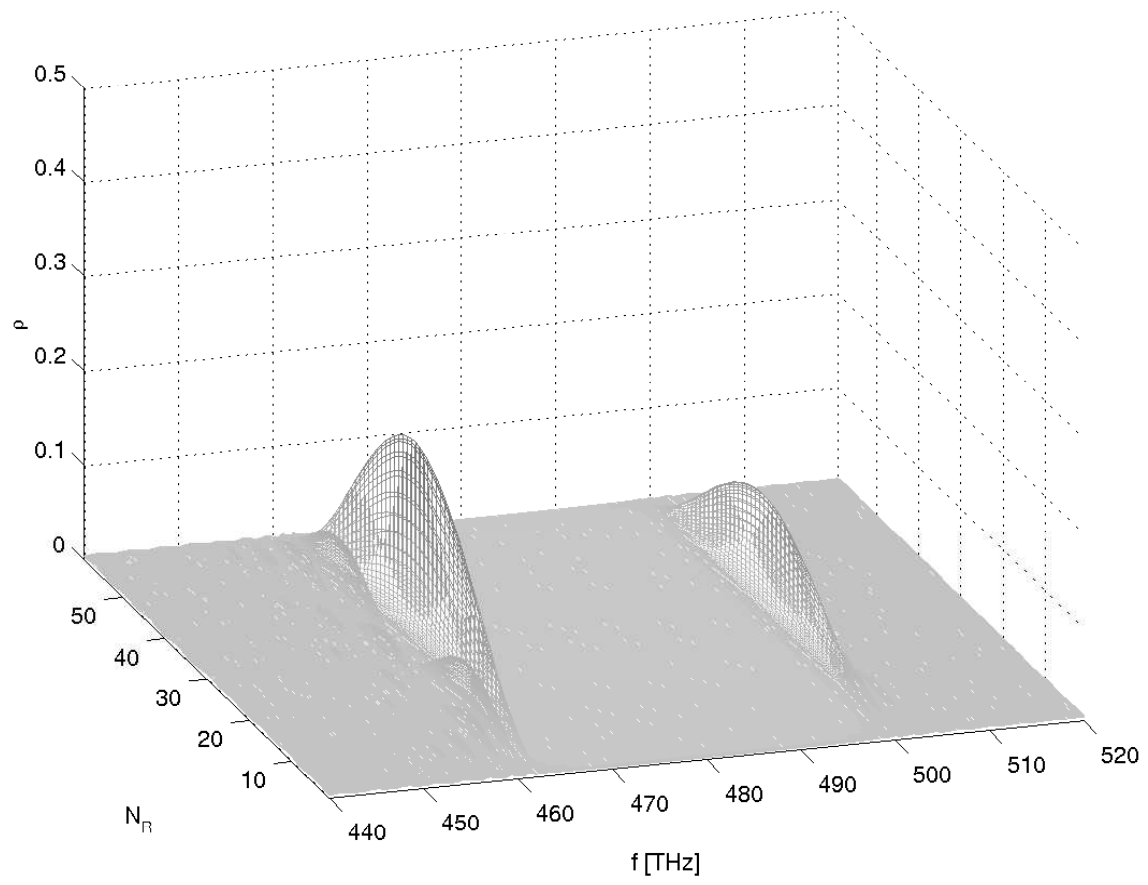

Figure 5. Mode spectrum in a 1D photonic crystal.

\section{REFERENCES}

1. E. Yablonovitch, "Inhibited spontaneous emission in solid-state physics and electronics," Phys. Rev. Lett. 58, p. 2059, 1987.

2. S. John, "Strong localization of photons in certain disordered dielectric superlattices," Phys. Rev. Lett. 58, p. 2486, 1987.

3. A. Rudziński, Problem of density of states of electromagnetic field in two-dimensional photonic crystals, Master's thesis, Warsaw University of Technology, 2004.

4. A. Rudziński, Problem of spontaneous emission process in photonic crystals, Bachelor's thesis, Warsaw University of Technology, 2003.

5. J. D. Jackson, Classical electrodynamics, J. Wiley, 1975.

6. C. H. P. Yeh, A. Yariv, "Electromagnetic propagation in periodic stratified media. i. general theory," J. Opt. Soc. Am. 67, p. 423, 1977.

\section{ACKNOWLEDGMENTS}

Project granted by the Ministry of Science and Information Society Technologies in Poland, project no 3 T11B 06928 and the Sixth European Union Framework Programme for Research and Technological Development (FP6) - Network of Excellence on Micro-Optics "NEMO". 